\title{
IDENTIFICAÇÃO DE PERFIL DE RISCO DE VULNERABILIDADE ACADÊMICA NO CURSO DE ENGENHARIA CIVIL DA UNIVERSIDADE FEDERAL DE ALAGOAS
}

Yanna Patriota de Souza Siqueira - yanna.siqueira@ctec.ufal.br Universidade Federal de Alagoas, Campus A. C. Simões Av. Lourival Melo Mota, S/N, Tabuleiro dos Martins 57072-900 - Maceió - Alagoas

Gabriela Silveira de Azevedo - gabriela.azevedo@ctec.ufal.br Universidade Federal de Alagoas, Campus A. C. Simões

Av. Lourival Melo Mota, S/N, Tabuleiro dos Martins 57072-900 - Maceió - Alagoas

Mateus Felipe Marques de Oliveira-mateus.oliveira@ctec.ufal.br Universidade Federal de Alagoas, Campus A. C. Simões Av. Lourival Melo Mota, S/N, Tabuleiro dos Martins 57072-900 - Maceió - Alagoas

Roberto Barbosa dos Santos - rbs@ctec.ufal.br Universidade Federal de Alagoas, Campus A. C. Simões Av. Lourival Melo Mota, S/N, Tabuleiro dos Martins 57072-900 - Maceió - Alagoas

Resumo: Mediante um cenário de evasão constante por parte dos alunos do ensino superior, o que é uma realidade nos cursos de Engenharia Civil, atrelado a um baixo desempenho acadêmico no decorrer do curso, debate-se acerca de perfis de risco, de um conjunto de alunos mais propícios a essa problemática. A partir de estudos, identificou-se um perfil de vulnerabilidade acadêmica: os estudantes ingressantes no segundo semestre letivo do curso de Engenharia Civil da Universidade Federal de Alagoas. Por meio de testes estatísticos, levantou-se possíveis causas dessa vulnerabilidade, às quais estão ligadas de forma direta com a entrada tardia de discentes no ensino superior. Causas essas que precedem de uma base escolar precária juntamente com uma falta de suporte a esses alunos. Diante desse cenário, propõe-se soluções viáveis e práticas com o apoio direto da unidade acadêmica atrelado a grupos discentes da própria universidade.

Palavras-chave: Vulnerabilidade. Evasão. Desempenho. Acadêmico(a). Estatística.

\section{INTRODUÇÃO}

Levando em consideração estudos recentes disponibilizados pelo Ministério de Educação e Cultura (MEC, 2018), o curso de Engenharia Civil se manteve entre os cursos mais procurados nos últimos anos. No entanto, apesar do elevado nível de competitividade para ingresso no curso, de acordo com pesquisas anteriores, o alto índice de evasão é encarado como realidade dentro deste, o que leva a acreditar que existem perfis de vulnerabilidade acadêmica que 
influenciam diretamente nesse índice, de maneira a sobrepor a vontade de muitos estudantes em concluir a graduação.

O curso de Engenharia Civil da UFAL foi implantado juntamente com a criação do Centro de Tecnologia (CTEC), anteriormente chamado de Escola de Engenharia, a qual teve seu funcionamento autorizado pelo Decreto No. 37.376 de 24 de Maio de 1955. Após aproximadamente 65 anos de história, há algumas mudanças consideráveis para objeto de estudo, como o Projeto Pedagógico do Curso (PPC) que, em 2006, registrou a mudança de regime anual para regime semestral, atendendo à reestruturação do regime acadêmico da Universidade. Como também, a inserção das Diretrizes Curriculares Nacionais, aprovadas em 2002, as quais foram renovadas em 2019 , de modo que o curso passa atualmente por um novo processo de reformulação.

Anualmente são ofertadas 80 vagas para a composição de duas turmas, primeiro e segundo semestre, com 40 alunos em cada. De acordo com o Manual do Candidato Ufal Sisu (2017), a composição das turmas é realizada obedecendo ao percentual de distribuição de vagas por demanda, bem como "seguindo rigorosamente à ordem de classificação das notas obtidas no Exame Nacional do Ensino Médio (ENEM) pelos alunos que realizaram a Pré-matrícula”.

Sendo assim, baseado no fato de que os ingressantes na universidade vivem realidades diferentes, as quais em diversas situações não os permitem formar a base de conhecimento necessária para o alcance de um bom desempenho nas fases iniciais do curso, é observado um ciclo de reprovações, desmotivação e abandono do curso ao longo dos anos. Ademais, nota-se uma tendência de representação de um grupo de vulnerabilidade acadêmica, que no cenário do Curso de Engenharia Civil da UFAL é a turma ingressante no segundo semestre letivo. Este perfil é caracterizado por uma entrada tardia na Universidade, se comparado com o ingresso da primeira turma, de pelo menos um período letivo. Acredita-se que essa lacuna temporal gera desmotivação, bem como afasta a prática de estudo contínuo, entre a saída do ensino médio e ingresso no ensino superior. Além disso, de acordo com o critério de composição de turmas, as notas obtidas no ENEM por esse perfil podem representar uma deficiência de base, amplamente falada em pesquisas e trabalhos anteriores, que pode refletir em uma maior dificuldade de aprendizagem para essa turma.

Diante desse contexto, o presente trabalho tem por objetivo identificar, por meio de análises estatísticas, se os alunos da segunda entrada do ano letivo se encaixam em um perfil de vulnerabilidade acadêmica, levando em consideração o modelo de ingresso atual. Para isso, a análise que será exposta tem como objeto de estudo os coeficientes de rendimento apresentados pelos alunos no primeiro período de graduação em Engenharia Civil da UFAL nos últimos dez anos, dividindo-os em dois grupos, tomando como critério para esta divisão os alunos pertencentes à primeira e segunda entrada pelo Sistema de Seleção Unificada (SISU), assim como uma análise do índice de evasão encontrado nessas turmas.

\section{REFERENCIAL TEÓRICO}

\subsection{Vulnerabilidade acadêmica}

De acordo com o Plano Nacional de Assistência Estudantil (PNAES), a vulnerabilidade acadêmica pode ser definida como o conjunto de fatores socioeconômicos, culturais, psicológicos, familiares, cognitivos e institucionais que fragilizam o discente perante o ambiente acadêmico e favorecem a sua evasão (BRASIL, 2010). 
Segundo a literatura, a vulnerabilidade no ensino superior surge a partir de diferentes causas, sendo elas associadas a aspectos de satisfação ou não da escolha, relações de dinâmica entre circunstâncias pessoais e características institucionais, como afirmam autores como Polydoro et. al (2001).

Outros autores, como Cerqueira (2003), Lehman (2005) e Ribeiro (2005), explanam em seus trabalhos motivos que corroboram com a existência da vulnerabilidade acadêmica no país, como os fatores relacionados às deficiências escolares pré-existentes e falta de apoio familiar; mau desempenho durante o curso, refletido em reprovações disciplinares; problemas financeiros; insatisfação geral com o curso e pouca integração social com a universidade pertencente.

\subsection{Evasão acadêmica}

Os elevados índices de evasão acadêmica retratam um problema que afeta as Instituições de Ensino Superior (IES) em âmbito nacional, conforme apresenta o Resumo Técnico do Censo da Educação Superior (MEC/INEP, 2009). Ainda de acordo com o Resumo, essa realidade aumenta ao passar dos anos no ambiente universitário.

Segundo a Comissão Especial de Estudos sobre a Evasão nas Universidades Brasileiras do Ministério da Educação (BRASIL, 1996, p. 18), há três tipos principais de evasão: evasão de curso, definida como a saída permanente do aluno do seu curso de origem, sem finalizá-lo; evasão da instituição, denominada como o desligamento do aluno da universidade em que está, havendo a possibilidade de troca de curso; e evasão do sistema de ensino superior, caracterizada como o afastamento pleno dos estudos universitários. Essas definições continuam atuais de acordo com estudos mais recentes, visto que a distinção dos tipos de evasão ajuda a compreender o fenômeno em sua complexidade, que decorre de diversos fatores já mencionados.

Conforme salientam pesquisas publicadas pelo Instituto Nacional de Estudos e Pesquisas Educacionais Anísio Teixeira (INEP, 2013), apenas 20\%, aproximadamente, de estudantes que ingressaram em cursos de graduação da área de conhecimento "Engenharia, Produção e Construção" chegaram a concluir o curso de graduação. Esse dado fomenta a ideia de que há uma preocupante tendência à evasão com ênfase em cursos de exatas, que exigem um certo grau de preparo e uma base matemática fundamentada.

Diante dessa conjuntura, como mostram estudos acerca da evasão nos cursos superiores, autores como Baggi e Lopes (2011) e Silva Filho et al. (2007) relacionam a evasão do ensino superior ao baixo desempenho escolar, à reprovação recorrente, ao abandono dos estudos e, consequentemente, a prejuízos nas políticas públicas, corroborando com os aspectos citados em outros trabalhos, descritos anteriormente, provenientes da vulnerabilidade acadêmica.

\section{METODOLOGIA}

Para o desenvolvimento do estudo foram definidas algumas etapas de trabalho, descritas a seguir:

\section{Coleta de dados}

Os dados contendo as notas em todas as disciplinas de todos os discentes do curso de Engenharia Civil da Universidade Federal de Alagoas do campus A. C. SIMÕES, no período que compreende os semestres letivos 2010.1 à 2019.2, foram 
obtidos mediante solicitação ao Núcleo de Tecnologia da Informação (NTI) da Universidade.

\section{Determinação dos grupos de estudo}

Optou-se pela divisão dos discentes em dois grupos, sendo o primeiro referente aos alunos que ingressaram na universidade no primeiro semestre, cursando todas as disciplinas referentes ao primeiro período do curso, obtendo nestas um dos seguintes conceitos: reprovado, reprovado por média ou aprovado. O segundo refere-se aos alunos que ingressaram na universidade no segundo semestre, seguindo as mesmas restrições.

\section{Determinação dos parâmetros de desempenho}

Para a verificação do rendimento dos alunos após seu ingresso na universidade para os dois grupos supracitados, calcularam-se os coeficientes de rendimento de cada aluno. Além disso, para a determinação do índice de evasão inerente aos grupos 1 e 2, calcularam-se os percentuais de evasão por semestre letivo.

\section{Cálculo do coeficiente de rendimento}

O coeficiente de rendimento foi calculado através da ponderação das notas obtidas nas disciplinas do primeiro semestre pela carga horária das mesmas, sendo as cargas horárias de cada disciplina apresentadas na Tabela 1.

Tabela 1 - Relação da carga horária das disciplinas referentes ao primeiro período do curso

\begin{tabular}{|c|c|}
\hline Disciplina & Carga horária (h) \\
\hline Cálculo 1 & 60 \\
\hline Desenho 1 & 60 \\
\hline Geometria Analítica & 60 \\
\hline Introdução à Computação & 60 \\
\hline Introdução à Engenharia & 30 \\
\hline Metodologia Científica & 30 \\
\hline
\end{tabular}

Fonte: Adaptado de PPC Engenharia Civil - UFAL (2006).

\section{Cálculo do índice de evasão}

Utilizaram-se os grupos mencionados na etapa II. No entanto, apenas aqueles que ingressaram até o ano letivo de 2015, que teriam tido oportunidade de concluir o curso até o ano letivo de 2019, respeitando o mínimo de 5 anos de graduação. O índice de evasão foi obtido calculando-se o percentual, por semestre letivo, do número de alunos que não se formaram, ou seja, que não foram aprovados ou não se matricularam até 2019.2 nas duas últimas disciplinas do curso.

\section{Teste de hipótese entre duas médias}

Com base na divisão proposta na etapa II, testaram-se duas hipóteses, utilizandose $\mathrm{o}$ teste $\mathrm{z}$ para médias independentes. A primeira para determinar se havia diferença entre as médias dos alunos que compõem os grupos 1 e 2, e a segunda para verificar se os alunos do grupo 1 possuem melhor rendimento no primeiro período do curso em relação aos alunos do grupo 2 . 
Analogamente, utilizou-se o teste t para a diferença entre médias independentes para verificar se havia diferença entre o índice de evasão dos alunos dos grupos 1 e 2 .

Os testes seguiram a formulação estatística proposta por Larson e Farber (2015) sendo as hipóteses nulas e alternativas para os testes, apresentadas nas Tabelas $2 \mathrm{e}$ 3.

Tabela 2 - Teste z para os coeficientes de rendimento dos grupos 1 e 2

\begin{tabular}{|l|c|c|}
\hline Teste 1 & H0: $\mu 1=\mu 2$ & Ha: $\mu 1 \neq \mu 2$ \\
\hline Teste 2 & H0: $\mu 1<=\mu 2$ & Ha: $\mu 1>\mu 2$ \\
\hline \multicolumn{2}{|c|}{ Fonte: Adaptado de Larson e Farber (2015). }
\end{tabular}

Tabela 3 - Teste t para os índices de evasão dos grupos 1 e 2

\begin{tabular}{|c|c|c|}
\hline Teste 3 & H0: $\mu 1=\mu 2$ & Ha: $\mu 1 \neq \mu 2$ \\
\hline
\end{tabular}

\section{RESULTADOS}

A partir do cálculo dos coeficientes de rendimento para os dois grupos, realizado na etapa IV, obtiveram-se amostras de tamanho 295 e 234 para os grupos 1 e 2, respectivamente.

Para o teste 1 , referente ao Teste $\mathrm{z}$, mencionado na etapa $\mathrm{V}$, visto tratar-se de um teste bicaudal, os valores críticos de $\mathrm{z}$ para um nível de significância de 5\% foram tomados como sendo $\mathrm{z}_{0}= \pm 1,96$ segundo a tabela de distribuição normal padrão. $\mathrm{O}$ valor de $\mathrm{z}$ para um nível de significância de 5\% foi calculado através da Equação 1.

$$
z=\frac{(\overline{x 1}-\overline{x 2})-(\mu 1-\mu 2)}{\sigma \overline{x 1}-\overline{x 2}}
$$

Onde: $\overline{x 1}-\overline{x 2}$ é a diferença entre as médias amostrais;

$\sigma \overline{x 1}-\overline{x 2}$ é o erro padrão associado à diferença das duas médias;

$\mu 1-\mu 2$ é a diferença entre as médias populacionais, admitida como nula.

Obteve-se, $\mathrm{z}=2,47$. Portanto, tendo em vista que $\mathrm{z}>\mathrm{z}_{0}$, têm-se evidências suficientes ao nível de significância de 5\% para rejeitar-se a hipótese nula, e, assim, as médias dos alunos do grupo 1 diferem das médias dos alunos do grupo 2.

Além disso, no que diz respeito ao teste 2 da etapa $\mathrm{V}$, por tratar-se de um teste unilateral à direita, para um nível de significância de $5 \%$ o valor crítico de $\mathrm{z}$ segundo a tabela de distribuição normal padrão é tal que $z_{0}=1,65$. Como o valor de z é constante e igual a 2,47 , têm-se evidências suficientes ao nível de significância de 5\% para rejeitar-se a hipótese nula. Sendo assim, o rendimento dos alunos do grupo 1 é superior ao rendimento dos alunos do grupo 2. Essa informação também é comprovada quando calcula-se a porcentagem de alunos com o coeficiente de rendimento maior ou igual a 7, por semestre letivo analisado neste trabalho, do 2010.1 à 2019.2, apresentado no Gráfico 1. Adotou-se a nota 7 como referência, tendo em vista que esta é a média do curso para que um aluno seja aprovado nas disciplinas em condições habituais. 
Gráfico 1: Correlação do coeficiente de rendimento maior ou igual a 7 dos grupos 1 e 2

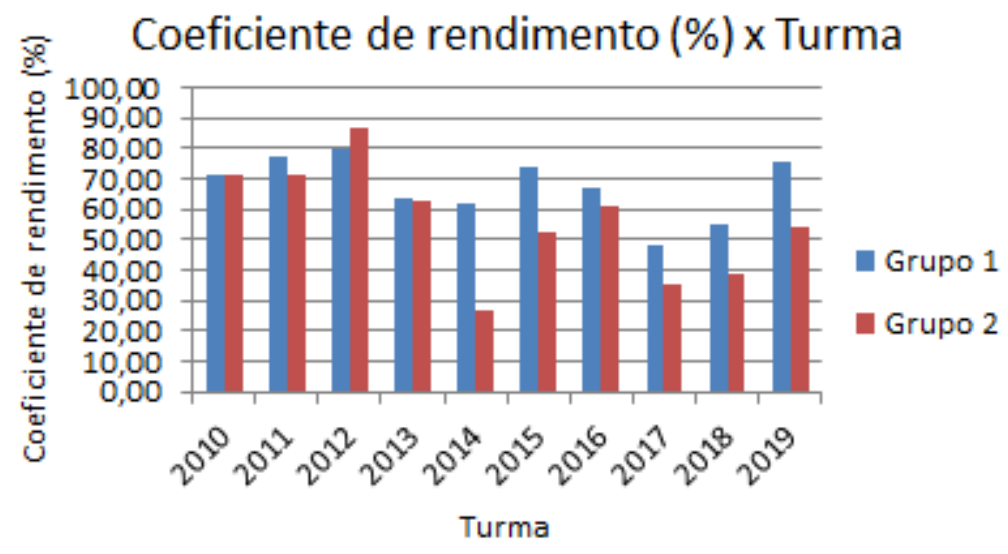

Fonte: Autores (2020).

A partir dessa verificação, constata-se que apenas em um ano letivo o grupo 2 superou o grupo 1, no ano de 2012. Em todos os demais, a porcentagem de alunos que obtiveram um coeficiente de rendimento maior ou igual a 7 do grupo 1 é igual ou superior à do grupo 2, o que ressalta a hipótese de que há uma tendência de vulnerabilidade do grupo 2 no que diz respeito ao desempenho acadêmico.

Em relação ao teste 3, referente ao Teste t, cuja hipótese está explicitada na Tabela 3, visto tratar-se de um teste bicaudal com variâncias populacionais desconhecidas e grau de liberdade igual a 4, para um nível de significância de 5\%, os valores críticos de t tabelados são tais que $\mathrm{t}_{0}= \pm 2,776$. $\mathrm{O}$ valor de $\mathrm{t}$ nas condições apresentadas acima foi calculado através da Equação 2.

$$
t=\frac{(\overline{x 1}-\overline{x 2})-(\mu 1-\mu 2)}{\sigma \overline{x 1}-\overline{x 2}}
$$

Obteve-se $\mathrm{t}=-0,7$. Logo, visto que $|\mathrm{t}|<\left|\mathrm{t}_{0}\right|$ não há evidências suficientes ao nível de significância de 5\% para rejeitar-se a hipótese nula e, portanto, os índices de evasão relacionados ao grupo 2 não diferem dos índices relacionados ao grupo 1, como retratado no Gráfico 2.

Gráfico 2: Correlação do índice de evasão das turmas dos grupos 1 e 2

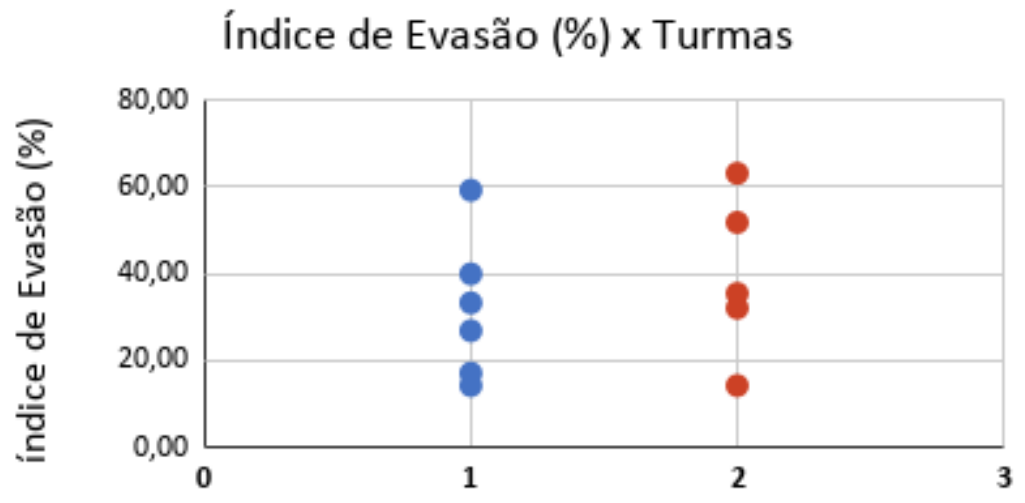

Grupos 1 e 2

Fonte: Autores (2020). 


\section{CONSIDERAÇÕES FINAIS}

A partir da análise obtida por meio dos resultados, constata-se que, no que diz respeito ao rendimento acadêmico dos recém-ingressos, levando-se em conta os grupos descritos na etapa II, os alunos que ingressam na universidade no primeiro semestre possuem melhor rendimento acadêmico comparado aos alunos que ingressam no segundo semestre, o que, por sua vez, caracteriza a entrada no segundo semestre como um perfil de vulnerabilidade acadêmica. Verifica-se assim uma das hipóteses iniciais de que uma menor nota no ENEM pode ser decorrente de uma base escolar mais defasada.

No que diz respeito aos índices de evasão decorrentes dos dois grupos estudados, a análise estatística mostrou-se inconclusiva, visto que não é possível determinar, ao nível de significância de 5\%, nenhuma diferença entre os grupos 1 e 2 .

Dessa forma, tendo em vista o perfil de vulnerabilidade acadêmica como sendo a turma do segundo semestre letivo, sugere-se a criação de um Curso de Verão por parte do setor administrativo do CTEC, com o objetivo de fornecer aos futuros ingressantes um contato direto e efetivo com a disciplina de matemática e seus conteúdos mais elementares. O Curso seria oferecido em um período anterior ao ingresso efetivo do segundo semestre, permitindo assim uma redução na lacuna de tempo em que estes discentes estão distantes da rotina de estudos. Dessa forma, pode-se reduzir os problemas de base, sanando algumas dificuldades nos conteúdos essenciais para disciplinas como Cálculo, Geometria Analítica e Introdução à Computação. Acredita-se que essa medida colabore com a redução da vulnerabilidade identificada.

Além disso, a fim de melhorar o índice de desempenho dos recém-ingressantes no curso, sugere-se também a criação de um Curso de Reforço voltado aos alunos do primeiro período, ministrado para cada turma, no turno oposto ao que os calouros do semestre de ingresso terão suas aulas obrigatórias da graduação. Nesse sentido, os alunos teriam a oportunidade de desenvolver melhor os assuntos aprendidos em sala de aula, de forma a focar não apenas nas dúvidas adquiridas, mas principalmente na fixação dos conteúdos por inteiro.

Sugere-se ainda uma participação ativa de grupos discentes da unidade como Centros Acadêmicos, Programas de Educação Tutorial e Programa de Capacitação Discente, na execução de ambos os cursos, tendo em vista que tais grupos visam a melhoria da graduação em engenharia civil como um todo, por meio de suas atividades.

\section{Agradecimentos}

Agradecemos de forma singular ao Programa de Educação Tutorial de Engenharia Civil da Universidade Federal de Alagoas pelo incentivo à prática de pesquisa.

\section{REFERÊNCIAS}

BAGGI, C. A. D. S.; LOPES, D. A. Evasão e avaliação institucional no ensino superior: uma discussão bibliográfica. Avaliação: Revista da Avaliação da Educação Superior, Curitiba, v. 16, n. 2, p. 355-374, 2011.

BRASIL. Decreto no 7.234, de 19 de julho de 2010. Dispõe sobre o Programa Nacional de Assistência Estudantil - PNAES. Brasília, Seção 1, p. 5., 2010. 
BRASIL. Decreto $n^{\circ} 37.376$, de 24 de maio de 1955. Dispõe sobre a autorização para funcionamento do curso de engenharia civil, da Escola de Engenharia de Alagoas.Brasília, Seção 1, p.10164, 1955.

BRASIL. Diplomação, retenção e evasão nos cursos de graduação em instituições de ensino superior públicas. Relatório da Comissão Especial de Estudos sobre Evasão nas Universidades Públicas Brasileiras. Brasília, SESu/MEC, p. 18., 1996.

BRASIL. Ministério da Educação e Cultura. Instituto Nacional de Estudos e Pesquisa Anísio Teixeira - INEP, 2013.

BRASIL. Ministério da Educação e Cultura. Instituto Nacional de Estudos e Pesquisa Anísio Teixeira - INEP, 2018.

CERQUEIRA, T. C. S. Evasão do curso de Pedagogia da UnB: A interpretação do aluno evadido. In: III Congresso Norte-Nordeste de Psicologia Construindo a Psicologia Brasileira: Desafios da ciência e prática psicológica, 2003, João Pessoa. Livro de resumos. vol II (pp.281283), João Pessoa, 2003.

FREITAS, Bruno. A.; COSTA, Erika. C. A. C.; COSTA, Cláudio. P. Fatores da evasão discente no curso de Engenharia Civil da Universidade Estadual da Paraíba. In: Congresso Brasileiro de Ensino em Engenharia, 44, 2016, João Pessoa Anais. Rio Grande do Norte, 2016.

LARSON, Ron; FARBER, Betsy. Estatística Aplicada. São Paulo: Pearson Education. 2015.

LEHMAN, Y. P. Estudo sobre a evasão universitária: As mudanças de paradigmas na educação e suas consequências. 2005. folhas. Tese de Livre Docência não publicada. Programa de Psicologia Social e do Trabalho, Universidade de São Paulo, São Paulo, 2005.

Manual do Candidato Ufal Sisu: Processo Seletivo 2017. COPEVE/PROGRAD, 2017.

MEC/INEP. Resumo Técnico: Censo da Educação Superior 2007. Brasília-DF. 2009. Disponível em: http://download.inep.gov.br/download/superior/censo/2007/Resumo_tecnico_2007.pdf. Acesso em: 7 jul. 2020.

POLYDORO, S. A. J., PRIMI, R., SERPA, M. N. F., ZARONI, M. N. H. e POMBAL, K. C. P. Desenvolvimento de uma escala de integração ao ensino superior. Avaliação: Psico-USF, v.6, p. 11-17, 2001.

POLYDORO, Soely Aparecida Jorge. O Trancamento de matrícula na trajetória acadêmica do universitário: condições de saída e de retorno à instituição. 2000. 179 f. Tese (Doutorado em Educação) - Programa de Pós-Graduação em Educação, Universidade Estadual de Campinas, Campinas, 2000. 
RANGEL, Flaminio. O. et al. Evasão e vulnerabilidade acadêmica em um curso de formação de professores de Ciências. In: IX Encontro Nacional de Pesquisa em Educação em Ciências, 2013, Águas de Lindóia. Atas. v. 9, Águas de Lindóia, 2013.

RIBEIRO, M. A. O projeto profissional familiar como determinante da evasão universitária: Um estudo preliminar. Revista Brasileira de Orientação Profissional, São Paulo, v. 6, n. 2, p. 55-70, 2005.

SILVA FILHO, R. L. L. et al. A evasão no ensino superior brasileiro. Cadernos de pesquisa, v. 37, n. 132, p. 641-659, 2007.

UFAL. Projeto Político Pedagógico do Curso de Graduação em Engenharia Civil Bacharelado. Maceió: UFAL, 2006. Disponível em: https://ufal.br/estudante/graduacao/projetos-pedagogicos/campus-maceio/ppc-engcivil.pdf/view. Acesso em: 15 jun. 2020.

\title{
IDENTIFICATION OF ACADEMIC VULNERABILITY RISK PROFILE IN THE CIVIL ENGINEERING COURSE OF THE FEDERAL UNIVERSITY OF ALAGOAS
}

\begin{abstract}
Through a scenario of constant evasion by students of higher education, which is a reality in the courses of Civil Engineering, linked to a low academic performance during the course, there is debate about risk profiles, of a set of students more prone to this problem. Based on studies, a profile of academic vulnerability was identified: students entering the second semester of the course of Civil Engineering at the Federal University of Alagoas. Through statistical tests, possible causes of this vulnerability were raised, to which they are directly linked with the late entry of students into higher education. Causes that precede a precarious school base together with a lack of support for these students. Because of this scenario, viable and practical solutions are proposed with the direct support of the academic unit linked to student groups in the university itself
\end{abstract}

Keywords: Vulnerability. Evasion. Performance. Academic. Statistics. 